\title{
О РАЗДЕЛЕННОМ РЕШЕНИИ СИСТЕМ УРАВНЕНИЙ УСТАНОВИВШИХСЯ РЕЖИМОВ ЭНЕРГЕТИЧЕСКИХ И ИНЖЕНЕРНЫХ СЕТЕЙ В СИСТЕМАХ УПРАВЛЕНИЯ
}

\author{
(Представил Ю. Яаксоо)
}

\section{1. Введение}

Современные энергетические и инженерные сети (ЭИС), такие как сети электро-, тепло-, водо- и газоснабжения, представляют собой сложные рассредоточенные объекты, состоящие из относительно автономных региональных подсистем. Их эксплуатация связана с решением ряда трудоемких системных задач (оценка состояния, моделирование режимов, оптимизация), выполняемым до сих пор на ЭВМ крупных центров диспетчерского управления, причем как исходные данные, так и результаты решения передаются по каналам связи.

Одно из главных направлений нынешнего этапа развития ЭИС заключается в создании децентрализованных систем управления (ДСУ) $\left[{ }^{1,2}\right]$, отличающихся: 1) высокой живучестью, 2) гибкостью использования вычислительных ресурсов, вытекающей из размещения ЭВМ у непосредственных потребителей вычислительных услуг, 3) рациональным использованием каналов связи за счет сокращения передачи данных локального характера. Переворот в строении систем управления порождает, вместе с тем, новые возможности решения общесистемных задач $\left[{ }^{3,4}\right]$. Размещение отдельных ЭВМ ДСУ в региональных центрах управления и/или в крупных узлах ЭИС позволяет выбирать между тремя способами их решения: 1) в полном объеме на одной ЭВМ, скорее всего на ЭВМ главного центра управления, 2) в виде независимых упрощенных задач на региональных ЭВМ, 3) в полном объеме на нескольких ЭВМ (разделенно), выделяя для каждой из них собственную подзадачу.

Ниже рассматривается последний, нетрадиционный способ решения, в частности, его применение к решению уравнений установившегося режима (УР), являющемуся одной нз более массовых задач при эксплуатации ЭИС. После постановки задачи УР на традиционной модели вводится понятие структурированной модели (СM), служащей основой ее разделенного решения. Далее рассматриваются два частных вида CM УР, представляемых системами уравнений блочно-диагональной с окаймлением (БДО) и блочно-трехдиагональонй (БТД) формы и предлагается ряд простых разделенных итерационных процессов на них. Обосновывается целесообразность регионального принципа структурирования. Наконец, приводится пример структурирования узловой модели ЭИС. 


\section{2. Задача моделирования УР эИС}

Математическое моделирование УР ЭИС сводится к формированию и решению совместной системы нелинейных уравнений электрической или гидравлической цепи

$$
F(z, \pi)=0,
$$

где $z=\{\hat{z}, \bar{z}\}, \hat{z}$ и $\bar{z}$ - множества искомых и заданных переменных, приписанных к координатам (узлам, контурам) цепи; $\pi$ - известные параметры ветвей цепи (схем замещения элементов ӘИС); $\operatorname{dim}(F)=$ $=c(\hat{z}), \operatorname{dim}-$ размерность, $c-$ кардинальное число. Конкретный вид системы (1) зависит от выбора координат и характера функций, аппроксимирующих характеристики элементов ЭИС. Для сетей электроснабжения она обычно имеет форму т. н. узловых уравнений, выражающих баланс активной и реактивной составляющих тока в узлах электрической цепи. Множество $z$ включает при этом модули и фазы узловых напряжений, а также активные и реактивные составляющие мощностей источников и стоков, подключенных к узлам цепи. Разделение его на подмножества искомых и заданных переменных зависит от конкретной постановки задачи [5]. Для трубопроводных сетей система (1) обычно имеет форму т. н. контурных уравнений [ $\left.{ }^{6}\right]$. Искомыми переменными $\hat{z}$ в ней являются контурные расходы (расходы в хордах графа гидравлической цепи), а заданными переменными $\bar{z}-$ производительности источников потока (расходы стоков) и напоры источников давления, подключенных соответственно к узлам и ветвям этой цепи.

\section{3. Структурированное представление модели УР}

Разделенное решение системы (1) требует ее преобразования в эквивалентную блочную систему разбивкой или прямым формированием по частям. Ограничимся рассмотрением частного типа полученных таким образом блочных систем, структура которых определяется расчленением ЭИС на непересекающиеся связные подсети. Специфика такой модели УР, называемой в дальнейшем структурированной, заключается в наличии в ней составляющих двух видов - блоков, которые описывают УР отдельных подсетей и т. н. блоков связи. В интересах простоты и конкретности изучим лишь те структурированные эквиваленты модели (1), вывод которых не влечет за собой расширения ее базиса переменных, и следовательно, повышения размерности.

Пусть ЭИС расчленена на связные подсети с индексами $k=1, \ldots, x$, определенные непересекающимися множествами узлов и ветвей моделирующей ее цепи. Соответствующая такому расчленению СМ может быть записана в виде

$$
F_{k}\left(z_{k}, z_{c k}^{\mathrm{r}}, \pi_{k}, \pi_{c k}^{\mathrm{r}}\right)=0, \quad k=1, \ldots, x,
$$

где $z_{k}, \pi_{k}$ - переменные и параметры $k$-й подсети; $z_{c k}^{\mathrm{r}}=\underset{l \in \operatorname{Adj}(k)}{U} z_{l k}^{\mathrm{r}}, z_{l k}^{\mathrm{r}}-$ переменные тех координат $l$-ой подсети, которые смежны с координатами $k$-й подсети, $\operatorname{Adj}(k)$ - множество, включающее индексы тех подсетей, которые имеют смежные координаты с $k$-й подсетью; $\pi_{c k}^{\mathrm{r}}=\underset{l \in \operatorname{Adj}(k)}{\bigcup} \pi_{l k}^{\mathrm{r}}, \pi_{l k}^{\mathrm{r}}-$ параметры $l$-й подсети, входящие в уравнения $k$-й подсети; $\operatorname{dim}\left(F_{k}\right)=c\left(z_{k}\right)$. Такие координаты и связанные с ними 
переменные $z_{k}^{\mathrm{r}}=\bigcup_{l \in \operatorname{Adj}(k)} z_{k l}^{\mathrm{r}}$, а также параметры $\pi_{k}^{\Gamma}=\underset{l \in \operatorname{Adj}(k)}{U} \pi_{k l}^{\mathrm{r}}$ усло-

вимся называть граничными для $k$-й подсети, остальные же координаты, переменные $z_{k}^{\mathrm{B}}=z_{k} \backslash z_{k}^{\mathrm{r}}$ и параметры $\pi_{k}^{\mathrm{B}}=\pi_{k} \backslash \pi_{k}^{\mathrm{r}}-$ внутренними. Для однородной системы координат можно СМ (2) получить подходящим пронумерованием переменных и упорядочением уравнений модели (1). Аналогичное структурирование ее для неоднородной системы координат может осуществляться, например, путем, описанным в $\left[{ }^{7}\right]$.

Конкретизуем СМ (2) для наиболее простых регулярных способов взаимосоединения подсетей, в частности, звездообразной и каскадной структуры их соединения. При первой существует одна центральная подсеть, которая связана со всеми остальными, не имеющими между собой непосредственных связей. Во второй, каждая подсеть, за исключением первой и последней, имеет по две соседних подсети.

3 вездообразное соединение подсетей. Приписывая центральной подсети индекс $k=x \quad$ с учетом $\operatorname{Adj}(x)=\{l / l=1, \ldots, x-1\}$, $\operatorname{Adj}(k)=\{x\}, k=1, \ldots, x-1, \quad$ приходим к следующему частному виду (2):

$$
\begin{aligned}
& G_{k}\left(z_{k}, z_{\varkappa}^{\mathrm{r}}, \pi_{k}, \pi_{\varkappa}^{\mathrm{r}}\right)=0, \quad k=1, \ldots, x-1, \\
& G_{\varkappa}\left(z_{\varkappa}, z_{1}^{\mathrm{r}}, \ldots, z_{\varkappa-1}^{\mathrm{r}}, \pi_{\varkappa}, \pi_{1}^{\mathrm{r}}, \ldots, \pi_{\varkappa-1}^{\mathrm{r}}\right)=0 .
\end{aligned}
$$

Эта система уравнений обладает структурой, аналогичной БДО-структуре систем линейных уравнений, которая, как выясняется ниже, является благоприятной с точки зрения установления сходимости одного вида блочных процессов итерации.

К аскадное соединение подсетей. В данном случае $\operatorname{Adj}(k)=$ $=\{k-1, k+1\}, k=2, \ldots, x-1 ; \operatorname{Adj}(1)=\{2\} ; \operatorname{Adj}(x)=\{x-1\}$ и $(2)$ превращается в

$$
\begin{aligned}
& D_{1}\left(z_{1}, z_{2}^{\mathrm{r}}, \pi_{1}, \pi_{2}^{\mathrm{r}}\right)=0, \\
& \dot{D}_{k}\left(z_{k}, z_{k-1}^{\mathrm{r}}, z_{k+1}^{\mathrm{r}}, \pi_{k}, \pi_{k-1}^{\mathrm{r}}, \pi_{k+1}^{\mathrm{r}}\right)=0, \\
& \dot{D}_{\varkappa}\left(z_{\varkappa}, z_{\varkappa-1}^{\mathrm{r}}, \pi_{\varkappa}, \pi_{\varkappa-1}^{\mathrm{r}}\right)=0 .
\end{aligned}
$$

Полученная система имеет структуру, аналогичную БТД-структуре систем линейных уравнений.

Заслуживает внимания, что получение СМ в виде БДО-системы не ограничено приведенным регулярным расчленением ЭИС. На самом деле ее можно вывести формально для любого соединения подсетей. Достаточно выделить из каждого блока модели (2) уравнения граничных координат и сосредоточить их в отдельный, $(x+1)$-й блок связи. Легко показать, что такая перегруппировка уравнений приводит к БДО-системе, состоящей из $x$ блоков уравнений внутренних координат для подсетей $1, \ldots, x$ и блока уравнений граничных координат:

$$
\begin{aligned}
& F_{k}\left(z_{k}^{\mathrm{B}}, z_{k}^{\mathrm{r}}, \pi_{k}^{\mathrm{BB}}, \pi_{k}^{\mathrm{Br}}\right)=0, \quad k=1, \ldots, x, \\
& F_{\mathrm{r}}\left(z^{\mathrm{r}}, z^{\mathrm{Br}}, \pi^{\mathrm{r}}, \pi^{\mathrm{Br}}, \pi^{\mathrm{Bn}}\right)=0 .
\end{aligned}
$$

Здесь $F_{k}$ и $F_{\text {г }}$ - вектор-функции размерностей $c\left(z_{k}^{\mathrm{B}}\right)$ и $\sum_{k=1}^{x} c\left(z_{k}^{\mathrm{r}}\right) ; z^{\mathrm{\Gamma}}=$ $=\bigcup_{k=1}^{\varkappa} z_{k}^{\mathrm{r}} ; z^{\mathrm{Br}}=\bigcup_{k=1}^{*} z_{k}^{\mathrm{Br}}, z_{k}^{\mathrm{Br}}-\quad$ подмножество внутренних переменных 
$k$-й подсети, входящих в уравнения граничных координат; $\pi^{\mathrm{r}}=\bigcup_{k=1}^{x} \pi_{k}^{\mathrm{r}}$, $\pi^{\mathrm{Br}}=\bigcup_{k=1}^{x} \pi_{k}^{\mathrm{Br}}, \pi^{\mathrm{BI}}=\bigcup_{k=1}^{x} \pi_{k}^{\mathrm{BI}} ; \pi_{k}^{\mathrm{BB}}, \pi_{k}^{\mathrm{Br}}$ и $\pi_{k}^{\mathrm{BI}},\left(\pi_{k}^{\mathrm{BB}} \cup \pi_{k}^{\mathrm{Br}} \cup \pi_{k}^{\mathrm{BI}}=\pi_{k}^{\mathrm{B}}\right)-$ некоторые непересекающиеся подмножества внутренних параметров $k$-й подсети, содержание которых зависит от выбранной системы координат. Переменные $z^{\mathrm{r}}$ можно, при этом, отнести к некоторой (в общем несвязной) стыковочной подсети, образующейся узлами и ветвями, связанными с граничными координатами. Наименьшую размерность блока (5б) получают, выбирая в качестве переменных связи лишь те переменные из $z^{\mathrm{r}}$, которые относятся к «развязывающим» координатам. Множество таких координат в узловой модели определяется наименьшим набором граничных узлов, удаление которых разделяет граф сети на $x$ несвязных подграфов подсетей $1, \ldots, x$. В контурной модели это действительно относительно дуального графа. Поскольку запись БДОсистемы в таком случае требует уточнения границ между подсетями, вывод ее приводится в качестве примера в п. 6 .

\section{4. Схемы разделенного итеративного решения}

Рассмотрим некоторые возможности построения процессов разделенного итеративного решения для систем типа (3) и (4). В интересах ясности будем пользоваться их менее детальной записью, опуская параметры, а также заданные переменные. Так, БДО-систему будем записывать в виде:

$$
\begin{aligned}
& G_{r}\left(w_{r}, w_{\rho}\right)=0, \quad r=1, \ldots, \varrho-1, \\
& G_{\rho}\left(w_{1}, \ldots, w_{\rho-1}, w_{\rho}\right)=0,
\end{aligned}
$$

причем для системы (3) $\varrho=\chi, w_{\rho}$ и $w_{r}$ - переменные центральной и остальных подсетей, а для системы (5) $\varrho=x+1, w_{\rho}-$ переменные граничных (развязывающих) координат $r$-й подсети; $\operatorname{dim}\left(G_{r}\right)=c\left(w_{r}\right)$, $\operatorname{dim}\left(G_{\rho}\right)=c\left(w_{\rho}\right)$. БТД-систему будем представлять сокращенно в виде:

$$
\begin{aligned}
& D_{1}\left(w_{1}, w_{2}\right)=0, \\
& \dot{D}_{r}\left(w_{r}, w_{r-1}, w_{r+1}\right)=0, \\
& D_{\rho}\left(w_{\rho}, w_{\rho-1}\right)=0,
\end{aligned}
$$

где $w_{r}, r=1, \ldots, \varrho-$ переменные $r$-ой подсети; $\operatorname{dim}\left(D_{r}\right)=c\left(w_{r}\right)$.

Допустим, что решение систем (6) и (7) проводится в сети из е вычислительных устройств (ВУ) с блоками памяти (БП), причем в БП $r$-го ВУ загружены лишь те данные, которые относятся к блоку уравнений, выделенному ему для решения.

На рис. 1 изображен обмен данными при разделенном решении системы (6). Само решение можно осуществить, например, следующим процессом блочной итерации:

$$
\begin{aligned}
& G_{r}\left(w_{r}^{n+1}, w_{\rho}^{n}\right)=0, \quad r=1, \ldots, \varrho-1, \\
& G_{\rho}\left(w_{1}^{n+1}, \ldots, w_{\rho-1}^{n+1}, w_{\rho}^{n+1}\right)=0, \\
& n=0,1, \ldots ; w_{\rho}^{0}=\bar{\zeta},
\end{aligned}
$$

где $\bar{\zeta}$ - начальное приближение вектора $w_{\rho}$. Каждое очередное приближение неизвестных $w_{1}, \ldots$, $w_{\rho}$ получают в два этапа вычислений, 
Рис. 1.

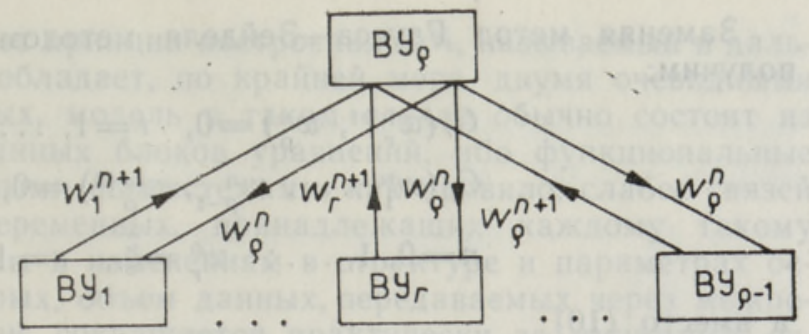

Сперва ВУ с индексами $r=1, \ldots$, Q-1 независимо и параллельно совершают один или несколько шагов итерации в каждом из блоков (8a) при фиксированном значении $w_{\rho}^{n}$. Вычисленные в частных итерационных процессах приближения $w_{r}^{n+1}$ затем передаются @-му ВУ, которое приводит итерацию (итерации) в блоке (8б) для определения $w_{\rho}^{n+1}$.

Процесс (8) реализует блочно-итеративный метод Гаусса-Зейделя с двумя блоками итерации $\left[{ }^{8}\right]$. Условия сходимости его при линейных уравнениях связи (6б) изучались в работе [ $\left.{ }^{9}\right]$. Если $G\left(w, w_{\rho}\right)=0$, $\mathrm{G}_{\rho}\left(w, w_{\rho}\right)=0$, где $G=\left\{G_{r} / r=1, \ldots, \varrho-1\right\}, w=\left\{w_{r} / r=1, \ldots, \varrho-1\right\}$ однозначно определяют неявные функции $w_{\rho}=g(w)$ и $w=g_{\rho}\left(w_{\rho}\right)$ соответственно, то процесс (8) равносилен процессу простой итерации

$$
\begin{aligned}
& w_{\rho}^{n+1}=g\left(g_{\rho}\left(w_{\rho}^{n}\right)\right)=\Gamma\left(w_{\rho}^{n}\right), \\
& n=0,1, \ldots ; w_{\rho}^{0}=\bar{\zeta} .
\end{aligned}
$$

Итак, можно установить условия сходимости процесса (9), а затем представить их в терминах исходного процесса (8). При этом необходимо учесть и те условия, выполнение которых гарантирует существование одноязычных функций $g(w)$ и $g_{\rho}\left(w_{\rho}\right)$.

C точки зрения временного выполнения процесс (8) квазипараллелен, так как оба упомянутых этапа вычислений связаны с двумя тактами работы сети ВУ: на первом, во время выполнения итераций в блоках $r=1, \ldots$, @-1, свободно $\varrho$-е ВУ, а на втором - все остальные.

В случае БТД-системы (7) процесс итерации типа Гаусса-Зейделя может организоваться следующим образом:

$$
\begin{aligned}
& D_{1}\left(w_{1}^{n+1}, w_{2}^{n}\right)=0, \\
& \dot{D}_{r}\left(w_{r}^{n+1}, w_{r-1}^{n+1}, w_{r+1}^{n}\right)=0, \\
& \dot{D}_{\rho}\left(w_{\rho}^{n+1}, w_{\rho-1}^{n+1}\right)=0 ; \\
& n=0,1, \ldots ; \quad w_{r}^{0}=\bar{\zeta}_{r}, \quad r=2, \ldots, \varrho-1 .
\end{aligned}
$$

Нетрудно показать, что в предположении симультанного решения блоков первое приближение значений $w_{1}, \ldots, w_{\rho}$ здесь достигается через @ тактов вычисления, а для каждого следующего приближения требуется лишь два такта. Соответствующая схема обмена данными изображена на рис. 2. На каждом четном такте могут работать ВУ с четными, а на каждом нечетном такте - с нечетными индексами.

Рис. 2.

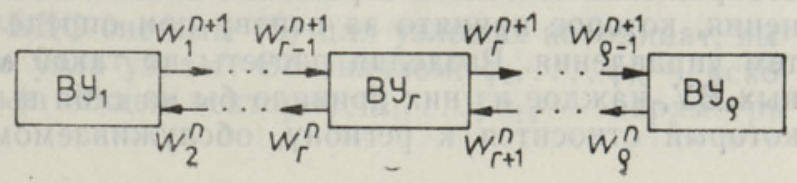



получим:

$$
\begin{aligned}
& G_{r}\left(w_{r}^{n+1}, w_{\rho}^{n}\right)=0, \quad r=1, \ldots, \varrho-1, \\
& G_{\rho}\left(w_{1}^{n}, \ldots, w_{\rho-1}^{n}, w_{\rho}^{n+1}\right)=0, \\
& n=0,1, \ldots ; \quad w_{r}^{0}=\bar{\zeta}_{r}, \quad r=1, \ldots, \varrho,
\end{aligned}
$$

а вместо (10):

$$
\begin{aligned}
& D_{1}\left(w_{1}^{n+1}, w_{2}^{n}\right)=0, \\
& \dot{D}_{r}\left(w_{r}^{n+1}, w_{r-1}^{n}, w_{r+1}^{n}\right)=0, \\
& \dot{D}_{\rho}\left(w_{\rho}^{n+1}, w_{\rho-1}^{n}\right)=0, \\
& n=0,1, \ldots ; \quad w_{r}^{0}=\bar{\zeta}_{r}, \quad r=1, \ldots \quad \varrho .
\end{aligned}
$$

Как в (11), так и в (12) требуется для вычисления каждого приближения значений неизвестных всего один такт, причем итерации во всех блоках могут проводиться параллельно.

Что касается решения самих блоков уравнений, то здесь могут применяться, в принципе, любые численные методы. При практическом их выборе должны учитываться конкретные условия - свойства блоков, требуемая точность решения, наличие хороших начальных приближений и др. При достаточно точных начальных приближениях, весьма часто имеющихся в эксплуатационном моделировании УР ЭИС, можно использовать методы типа Ньютона-Рафсона. В противном же случае следует прибегать к методам, менее требовательным к начальному приближению, базирующимся, например, на принципе вариации параметра $\left[{ }^{5,8}\right]$.

Блочно-итеративные методы придают решению значительную гибкость, предоставляя возможность независимого проведения внутриблочных процессов итерации с применением для отдельных блоков разных численных методов, чисел шагов итерации, коэффициентов релаксации. Подбор для блоков индивидуальных, наиболее подходящих численных методов смягчает остроту проблемы реальной сходимости процесса решения.

Приведенные итерационные схемы являются лишь некоторыми примерами сочетания CM с блочными методами итераций на них. Структурные особенности модели и конфигурация сети ВУ вместе со способами организации итеративных процессов, в том числе асинхронных $\left[{ }^{10}\right]$, и с различными численными методами порождают большое количество возможных процедур решения.

\section{5. Региональный принцип построения СМ}

Практическое построение СМ требует учета определенных условий, выполнение которых обеспечивало бы приемлемые характеристики расчетов, осуществляемых на ней, а также возможно малый объем обмениваемой при этом координирующей информации. Одно естественное соображение состоит в отражении в СМ того же регионального расчленения, которое принято за основу при определении локальных подсистем управления. Разделяя расчеты по такой модели в сети региональных ВУ, каждое из них приняло бы на себя вычисления в том ее блоке, который относится к региону, обслуживаемому им при выполнении 
функций управления. Этот принцип построения СМ, называемый в дальнейшем региональным, обладает, по крайней мере, двумя очевидными достоинствами. Во-первых, модель в таком случае обычно состоит из относительно слабосвязанных блоков уравнений, ибо функциональные связи между региональными подсистемами, как правило, слабее связей внутри их. Значения переменных, принадлежащих каждому такому блоку, малочувствительны к изменениям в структуре и параметрах остальных блоков. Во-вторых, объем данных, передаваемых через межрегиональные каналы связи, сокращается практически до минимума, ограничиваясь сравнительно малым количеством приближений для значений переменных связи. Минимизация объема обмена тем более существенна, чем чаще выполняются расчеты. Структурируя модель каким-то иным способом, неизбежна межрегиональная передача некоторой части исходных данных и результатов, а также увеличение количества переменных связи.

Реализацию описанного принципа усложняют ограничения вычислительного и/или коммуникативного характера. Высокая загруженность или недостаточная производительность отдельных ВУ, малая пропускная способность некоторых каналов связи и другие обстоятельства могут вводить коррективы в окончательный вид СМ, заставляя отказаться от чисто регионального принципа ее построения.

Частные реализации СМ отличаются числом и размерностью блоков уравнений, числом и содержанием переменных связи. Влияние этих характеристик CM на их вычислительные качества, также как быстрота сходимости итерационного процесса и временные затраты на одну итерацию, до сих пор недостаточно изучено. Некоторые оценки их зависимости от числа и порядка блоков содержатся в работе $\left[{ }^{11}\right]$ и в исследованиях, посвященных блочно-последовательному моделированию электроэнергетических систем и электронных схем $\left[{ }^{12}\right]$.

\section{6. Иллюстративный пример}

Приведем пример построения СМ для ЭИС с расчетной схемой, изображенной на рис. 3. Ветви схемы соответствуют элементам моделирующей электрической или гидравлической цепи, узлы - точкам их соединения, а стрелочки - источникам (генераторы, насосы) или стокам (потребители и их группы). Отделенные штриховой линией части схемы изображают подсети 1 и 2 , причем узел 4 и ветвь 7 принадлежат к 1-й, а узел 7 и ветвь 8 - ко 2 -й подсети.

Рис. 3.

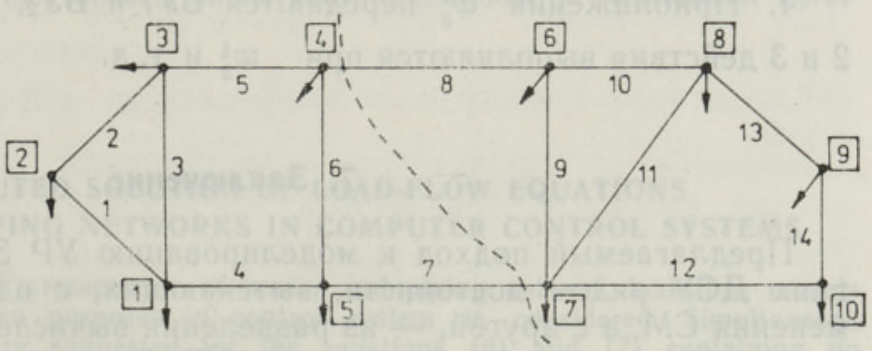

Выводим СМ в форме БДО-системы (5) для узловых координат, выбирая в качестве базового узла узел 1 . Обозначаем: $\varphi_{2}, \ldots, \varphi_{10}$ - искомые узловые переменные (в общем векторы), $\alpha_{1}, \ldots, \alpha_{14}$ - параметры ветвей. 
Тогда $z_{1}=\left\{\varphi_{2}, \ldots, \varphi_{5}\right\}, z_{2}=\left\{\varphi_{6}, \ldots, \varphi_{10}\right\} ; \pi_{1}=\left\{\alpha_{1}, \ldots, \alpha_{7}\right\}, \pi_{2}=$ $=\left\{\alpha_{8}, \ldots, \alpha_{14}\right\} ; \quad z_{1}^{\mathrm{r}}=\left\{\varphi_{4}, \varphi_{5}\right\}, \quad z_{2}^{\mathrm{r}}=\left\{\varphi_{6}, \varphi_{7}\right\} ; \pi_{1}^{\mathrm{r}}=\alpha_{7}, \pi_{2}^{\mathrm{r}}=\alpha_{8} ; \quad$ следовательно, $z_{1}^{\mathrm{B}}=\left\{\varphi_{2}, \varphi_{3}\right\}, z_{2}^{\mathrm{B}}=\left\{\varphi_{8}, \varphi_{9}, \varphi_{10}\right\} . \quad$ С учетом специфики узловой модели $\quad z_{1}^{\mathrm{Br}}=\varphi_{3}, \quad z_{2}^{\mathrm{Br}}=\left\{\varphi_{8}, \varphi_{10}\right\} ; \quad \pi_{1}^{\mathrm{BB}}=\left\{\alpha_{1}, \alpha_{2}, \alpha_{3}\right\}, \quad \pi_{2}^{\mathrm{BB}}=\left\{\alpha_{13}, \alpha_{14}\right\} ; \quad \pi_{1}^{\mathrm{Br}}=$ $=\left\{\alpha_{4}, \alpha_{5}\right\}, \pi_{2}^{\mathrm{Br}}=\left\{\alpha_{10} \alpha_{11}, \alpha_{12}\right\} ; \pi_{1}^{\mathrm{BI}}=\alpha_{6}, \pi_{2}^{\mathrm{B \Pi}}=\alpha_{9}$. Итак, узловая модель имеет вид:

$$
\begin{aligned}
& F_{1}\left(\varphi_{2}, \varphi_{3} ; \varphi_{4}, \varphi_{5} ; \alpha_{1}, \alpha_{2}, \alpha_{3} ; \alpha_{4}, \alpha_{5}\right)=\bar{\beta}_{1}, \\
& F_{2}\left(\varphi_{8}, \varphi_{9}, \varphi_{10} ; \varphi_{6}, \varphi_{7} ; \alpha_{13}, \alpha_{14} ; \alpha_{10}, \alpha_{11}, \alpha_{12}\right)=\bar{\beta}_{2}, \\
& F_{\Gamma}\left(\varphi_{4}, \ldots, \varphi_{7} ; \varphi_{3}, \varphi_{8}, \varphi_{10} ; \alpha_{7}, \alpha_{8} ; \alpha_{4}, \alpha_{5}, \alpha_{10}, \alpha_{11}, \alpha_{12} ; \alpha_{6}, \alpha_{9}\right)=\bar{\beta}_{\mathrm{r}},
\end{aligned}
$$

причем точками с запятой отделены множества переменных и параметров, $\bar{\beta}_{1}, \bar{\beta}_{2}$ и $\bar{\beta}_{\text {r }}$ обозначают векторы правых частей блоков уравнений.

Блок связи (13б) состоит из уравнений всех граничных узлов обеих подсетей. Выбираем среди этих узлов некоторый минимальный набор развязывающих узлов, например, 4 и 7 . После перенесения уравнений всех граничных узлов, кроме 4 и 7 , в блоки соответствующих подсетей имеем:

$$
\begin{aligned}
& F_{1}^{\prime}\left(\varphi_{2}, \varphi_{3}, \varphi_{5} ; \varphi_{4}, \varphi_{7} ; \alpha_{1}, \alpha_{2}, \alpha_{3} ; \alpha_{4}, \alpha_{5} ; \alpha_{6}, \alpha_{7}\right)=\bar{\beta}_{1}^{\prime}, \\
& F_{2}^{\prime}\left(\varphi_{6}, \varphi_{8}, \varphi_{9}, \varphi_{10} ; \varphi_{4}, \varphi_{7} ; \alpha_{13}, \alpha_{14} ; \alpha_{10}, \alpha_{11}, \alpha_{12} ; \alpha_{8}, \alpha_{9}\right)=\bar{\beta}_{2}^{\prime}, \\
& F_{\Gamma}^{\prime}\left(\varphi_{4}, \varphi_{7} ; \varphi_{3}, \varphi_{5}, \varphi_{6}, \varphi_{8}, \varphi_{10} ; \alpha_{7}, \alpha_{8} ; \alpha_{5}, \alpha_{11}, \alpha_{12} ; \alpha_{6}, \alpha_{9}\right)=\bar{\beta}_{\Gamma}^{\prime} .
\end{aligned}
$$

Описываем ход решения системы (14) по схеме блочной итерации (8), предполагая, что решение разделяется между ВУ ${ }_{1}, \mathrm{BУ}_{2}$ и координирующим $\mathrm{BУ}_{3}$, производящим расчеты по блокам $(14 a)$, (14б) и (148) соответственно.

1. Задаются начальные приближения переменных связи $w_{3}^{0}=\bar{\zeta}=\left(\varphi_{4}^{0}, \varphi_{7}^{0}\right\}$, значения их передаются ВУ 1 и ВУ ${ }_{2}$.

2. Выполняются независимые итерации (один или несколько шагов) по $(14 a)$ и (14б) в ВУ $w_{2}^{1}=\left\{\varphi_{6}^{1}, \varphi_{8}^{1}, \varphi_{9}^{1}, \varphi_{10}^{1}\right\}$.

3. Приближения $w_{1}^{1}$ и $w_{2}^{1}$ передаются ВУ ${ }_{3}$, выполняются один или несколько шагов по (148) для получения $w_{3}^{1}=\left\{\varphi_{4}^{1}, \varphi_{7}^{1}\right\}$.

4. Приближения $w_{3}^{1}$ передаются $\mathrm{BУ}_{1}$ и $\mathrm{BУ}_{2}$, описанные в разделах 2 и 3 действия выполняются при $w_{3}^{1}$ и т. д.

\section{7. Заключение}

Предлагаемый подход к моделированию УР ЭИС обладает в условиях ДСУ рядом достоинств, вытекающих, с одной стороны, из применения СМ, а с другой, - из разделения вычислений:

1) возможность моделирования УР больших ЭИС при малых единичных мощностях ВУ;

2) удобство, оперативность и надежность внесения в модель коррективов, касающихся изменений в структуре и параметрах подсетей;

3) значительные гибкость и автономность решения модельных блоков уравнений, относящихся к отдельным подсетям; 
4) сокращение объема данных, передаваемых по межрегиональным каналам связи. Это достоинство, как и отмеченное в разделе 2, проявляется наиболее явным образом при таком способе разделенного моделирования, где СМ формируется согласно региональному расчленению ЭИС, принятому в ДСУ;

5) ориентированность на разделенные базы данных.

\section{ЛИТЕ РА Т У Р А}

1. Кулик М. Н. // Электронное моделирование, 1983, № 6, 76-78.

2. Dillon, T. // Electrical Power and Energy Syst., 1985, 7, № 2, 67-74.

3. Happ, H., Undrill, J. // IEEE Trans, Power Appar. and Syst., 1969, № 6, 789-796.

4. Seidu, K., Mukai, H. // IEEE Trans. Power Appar. and Syst., 1985, № 5, 1026-1034.

5. Жуков Л. А., Стратан И. П. Установившиеся режимы сложных электрических сетей и систем. М., Энергия, 1979.

6. Евдокимов А. Г., Дубровский В. В., Тевящев А. Д. Потокораспределение в инженерных сетях. М., Стройиздат, 1979.

7. Wu, F. F. // IEEE Trans. Circuits and Syst., 1976, № 12, 706-713.

8. Ортега Дж., Рейнболдт В. Итерационные методы решения нелинейных систем уравнений со многими неизвестными. М., Мир, 1975.

9. Роозе А. // Изв. АН ЭССР. Физ. Матем., 1978, 27, № 2, 227-229.

10. Baudet, G. M. // J. of the ACM, 1978, № 2, 226-244.

11. Котарова Н. Н., Шамаева О. Ю. // Кибернетика, 1979, № 1, 112-119.

12. Борзенков Б. И., Малюк В. Г., Огороднейчук И. Ф. // Изв. вузов. Радиоэлектроника, 1984, № 10, 37-41.

Ннститут кибернетики

Академии наук Эстонской ССР

\section{Поступила в редакцию}

$12 / \mathrm{I} 1988$

\section{H. PEDAK}

\section{ENERGIA- JA INSENERIVORKUDE PUSIREZIIMIVORRANDITE JAOTATUD LAHENDAMISEST JUHTIMISSOSTEEMIDES}

Artiklis on käsitletud energia- ja insenerivõrkude püsirežiimimudelite ositiesitust (struktuurimist) võimaldamaks jaotada modelleerimisarvutusi hajusjuhtimissüsteemi raalide vahel. On pakutud mitmeid simultaanseid plokk-iteratsioonimeetodeid lisamuutujaid mittesisaldavate struktuuritud mudelikujudega (6) ja (7) opereerimiseks ning põhjendatud vōrgu juhtimisotstarbelist regionaalseteks alamvôrkudeks liigendamist kopeeriva struktuurimise otstarbekust lahendamise käiku koordineeriva andmevahetuse seisukohalt. Toodud näide illustreerib nn. sōlmevõrrandite ositi formeerimist ja lahendamist iteratsiooniskeemi (8) abil.

\section{H. PEDAK}

\section{ON DISTRIBUTED SOLUTION OF LOAD-FLOW EQUATIONS OF POWER AND PIPING NETWORKS IN COMPUTER CONTROL SYSTEMS}

Piecewise (partitioned) presentation of power and piping network load-flow models for distributive computation purposes in control system is considered. Simultaneous block iteration methods are suggested for the equations (6) and (7) containing no additional variables. Advantages of such partition, which follows the pattern used for control area assignment, are justified from the standpoint of data exchange. An example to illustrate distributive formation and solution of so-called node equations is given. 